\title{
Monophasic action potentials in a patient with multiform ventricular tachycardia without QT prolongation
}

\author{
Tetsuro Emori, Tohru Ohe, Katsuro Shimomura
}

\begin{abstract}
A 41 year old woman had multiform ventricular tachycardia without QT prolongation. Monophasic action potentials were recorded from the right ventricle during the attacks of multiform ventricular tachycardia and effective refractory periods were examined at the same sites. There was no abnormal hump to suggest early afterdepolarisation in the monophasic action potentials, but there was dispersion of the effective refractory period in the right ventricle $(80 \mathrm{~ms})$. Stimulation from the right ventricular apex, where the effective refractory period was shortest, reproducibly induced multiform ventricular tachycardia. Two weeks after admission, when her condition was stable, multiform ventricular tachycardia could not be induced and the dispersion of the effective refractory period in the right ventricle was $20 \mathrm{~ms}$.
\end{abstract}

(Br Heart f 1993;69:356-358)

Multiform ventricular tachycardia can be divided into two types according to the presence or absence of QT prolongation. Multiform ventricular tachycardia with QT prolongation is usually called torsades de pointes. ${ }^{1}$ Many investigators have emphasised the role of a repolarisation abnormality in torsades de pointes. ${ }^{23}$ However, the role of a repolarisation abnormality in multiform ventricular tachycardia without QT prolongation is unknown.

We report a patient with multiform ventricular tachycardia without QT prolongation. Monophasic action potentials were recorded from the right ventricle during the attacks of multiform ventricular tachycardia but showed no repolarisation abnormality. There was dispersion of the effective refractory period in the right ventricle caused by local shortening.

Division of

Cardiology,

Department of

Medicine, National

Cardiovascular

Centre, Suita, Osaka,

Japan

T Emori

T Ohe

K Shimomura

Correspondence to:

Dr Tetsuro Emori, Division of Cardiology, Department of Medicine, National

Cardiovascular Cen tre, 5-7 1 Fujishiro-dai, Suita, Osaka, Japan 565

\section{Case report}

A 41 year old woman with a history of several syncopal attacks was admitted to our hospital in 1980. The only abnormality detected was $R$ on $T$ type ventricular extrasystoles. The cause of the syncopal attacks was not discovered and she was followed up as an outpatient for 10 years without syncopal attacks.
In 1990 she was readmitted after several episodes of syncope. On this admission, multiform ventricular tachycardia was often recorded after $R$ on $T$ type ventricular extrasystoles (fig $1 \mathrm{~A}$ and $\mathrm{B}$ ). The electrocardiograms showed no ST change suggesting ischaemia and the QT interval was normal (the QT interval in lead II was $0.36 \mathrm{~s}$ and the QTc interval was $0.44 \mathrm{~s}$ ) (fig $1 \mathrm{C}$ ). The QRS configuration of the ventricular extrasystole
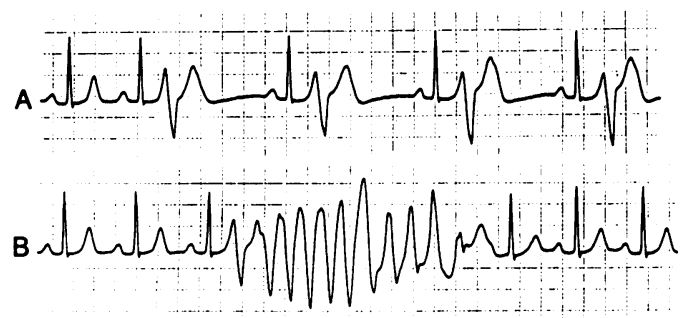

C

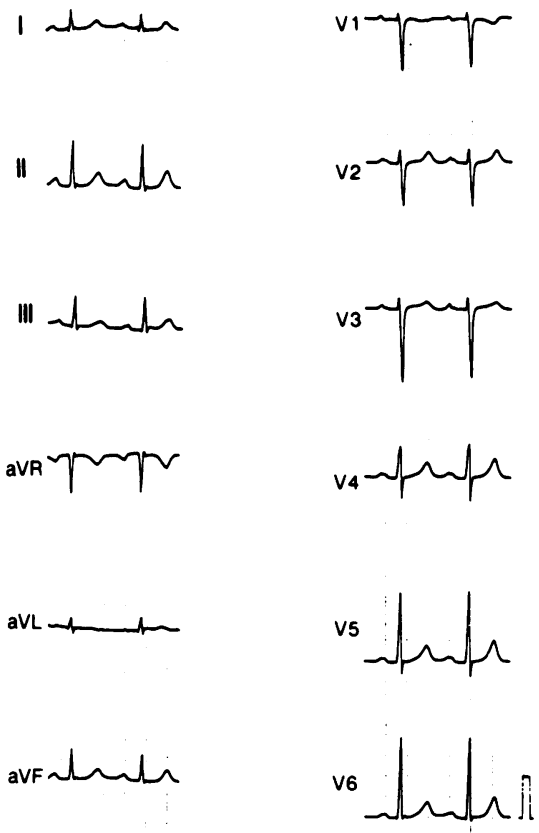

Figure 1 Tracings $A$ and $B$ are from monitor leads. $(A)$ extrasystoles with short coupling intervals. (B) Nonsustained multiform ventricular tachycardia occurred after the ventricular extrasystoles. (C) The electrocardiogram on admission did not indicate $Q T$ prolongation (the $Q T$ interval in lead II was $0.36 \mathrm{~s}$ and the QTC interval was $0.44 \mathrm{~s}$ ) or $S T$ changes suggesting ischaemia. The cardiac rhythm shows bigeminy of ventricular 
was a left bundle branch block with left axis deviation. Blood tests were normal except for hypokalaemia $(3 \cdot 2 \mathrm{mmol} / \mathrm{l})$. Monophasic action potentials were recorded to evaluate a repolarisation abnormality during the attacks of multiform ventricular tachycardia. Monophasic action potentials were recorded at the right ventricular apex, the right ventricular free wall, and the right ventricular septum during atrial pacing at a cycle length of $500 \mathrm{~ms}$. Effective refractory periods were examined at the same sites at a basic cycle length of $500 \mathrm{~ms}$. There was no abnormal hump in the monophasic action potential to

V5
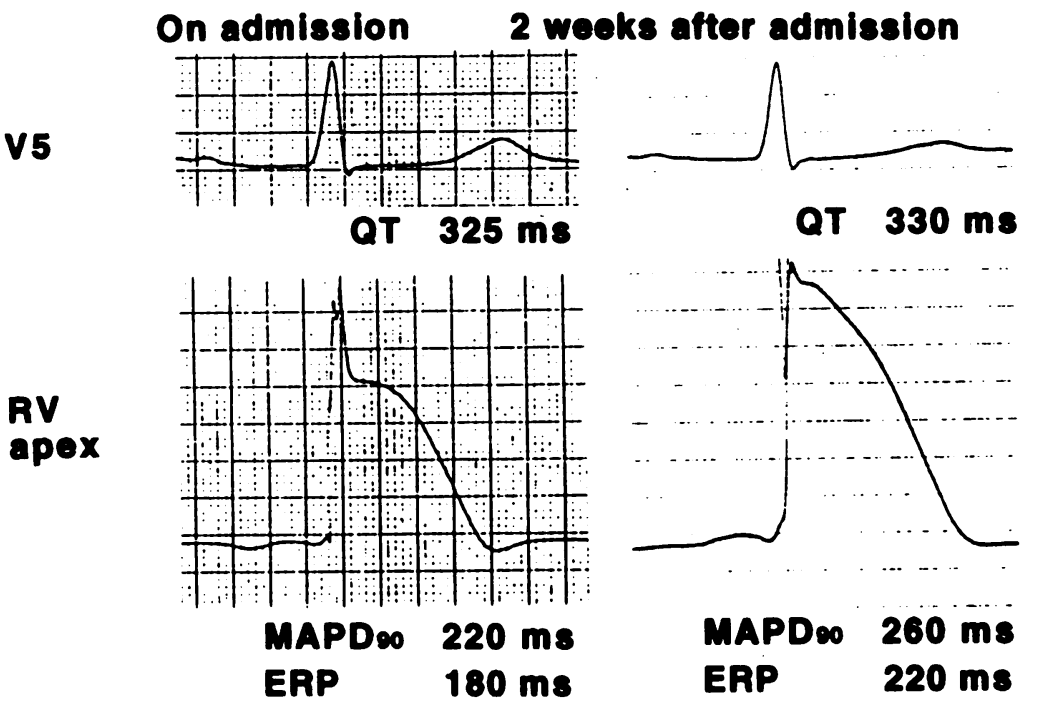

MAPDeo $180 \mathrm{~ms}$

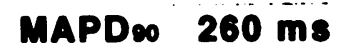
ERP $220 \mathrm{~ms}$
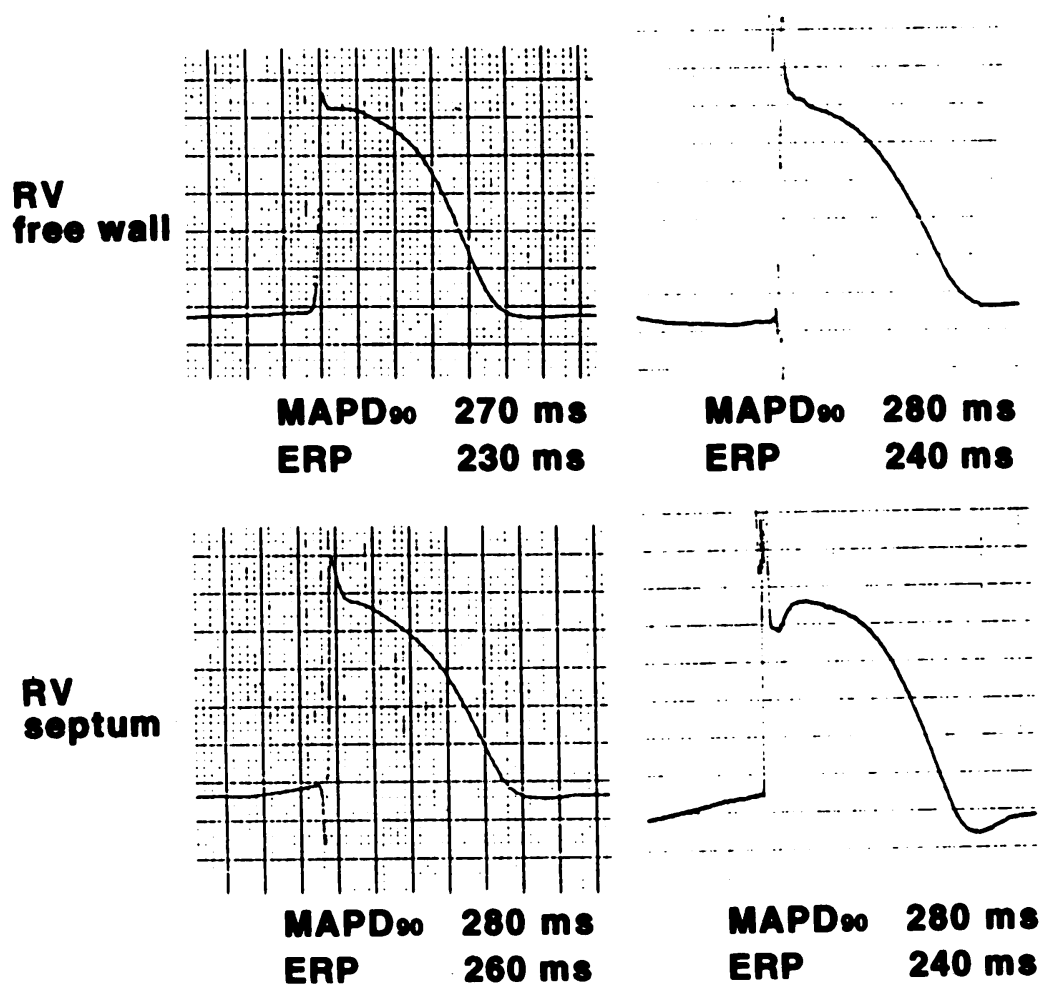

Figure 2 Monophasic action potentials obtained from the right ventricle. All recordings were obtained during atrial pacing at a cycle length of $500 \mathrm{~ms}$. The QT interval in lead $V 5$ was $325 \mathrm{~ms}$ on admission and $330 \mathrm{~ms}$ in the follow up study 2 weeks after admission. No abnormal hump indicating early afterdepolarisation was seen in any recording. However, the monophasic action potential duration and the effective refractory period at the right ventricular apex were shorter than those of the free wall or the midseptum on admission and there was dispersion of the effective refractory period in the right ventricle $(80 \mathrm{~ms})$. Two weeks later the dispersion of the effective refractory period had decreased to $20 \mathrm{~ms}$. RV, right ventricular; $M A P D_{90}$, monophasic action potential duration at $90 \%$ of the amplitude; ERP, effective refractory period. suggest early afterdepolarisation at any site (fig 2). Figure 2 shows the individual values of the monophasic action potential duration (the monophasic action potential duration at $90 \%$ of the amplitude was used), the effective refractory period at each site, and the QT interval in lead V5. The dispersion of the effective refractory period (maximum effective refractory period minus minimum effective refractory period) in the right ventricle was $80 \mathrm{~ms}$. A single paired extrastimulus from the right ventricular apex reproducibly induced multiform ventricular tachycardia. Multiform ventricular tachycardia could not be induced from the right ventricular free wall or the right ventricular septum, even by triple paired extrastimuli. Treatment with disopyramide infusion and potassium supplementation was started. Multiform ventricular tachycardia and the ventricular extrasystoles disappeared two days after admission. Echocardiography, myocardial scintigraphy, magnetic resonance imaging, and signal averaged electrocardiograms were normal. Neither multiform ventricular tachycardia nor abnormal QT prolongation could be induced by exercise testing or isoprenaline (isoproterenol) infusion. Electrophysiological study was repeated two weeks after admission, when the serum potassium concentration was normal $(4.0 \mathrm{mmol} / \mathrm{l})$. Disopyramide was stopped for three days before the study. Multiform ventricular tachycardia was not induced by stimulation at the right ventricular apex. The dispersion of the effective refractory period decreased from $80 \mathrm{~ms}$ to $20 \mathrm{~ms}$ (fig 2). Figure 2 shows the individual values of the monophasic action potential duration, the effective refractory period at each site, and the QT interval in lead V5.

\section{Discussion}

A repolarisation abnormality is regarded as an important cause of multiform ventricular tachycardia with QT prolongation. Early afterdepolarisations are especially important in the genesis of this arrhythmia. ${ }^{4-6}$ Monophasic action potentials were useful in the evaluation of a repolarisation abnormality, ${ }^{7}$ and early afterdepolarisations were recorded in patients with torsades de pointes associated with antiarrhythmic drugs, ${ }^{8}$ bradycardia, ${ }^{9,10}$ and congenital long QT syndrome. ${ }^{112}$ There have been no reports, however, on monophasic action potentials in patients with multiform ventricular tachycardia without QT prolongation. In the present study we were able to record monophasic action potentials during both the acute phase and the stable period, but we did not detect an abnormal hump in the monophasic action potentials that suggested early afterdepolarisations.

Kuo et al induced dispersion of the monophasic action potential durations in the ventricle (they defined "dispersion" as differences in the durations of monophasic action potentials in different portions of the ventricles) by a combination of general hypother- 
mia and selective coronary artery perfusion with warm blood in anaesthetised dogs and they also induced ventricular fibrillation during stimulation at the site with the shortest monophasic action potential duration. ${ }^{13}$ In this experiment dispersion of repolarisation was caused by shortening of the monophasic action potential duration in the selectively perfused area and its prolongation in the nonperfused area. Ventricular fibrillation was induced from only the area at which the monophasic action potential duration was short. They assumed that when the increase in dispersion of repolarisation reached a critical level, propagation of extrasystoles originating from the area with a short monophasic action potential duration encountered a block in the area with a long monophasic action potential duration and created conditions favourable for reentry.

Similarly, multiform ventricular tachycardia could be induced reproducibly in the present patient by stimulation at the right ventricular apex, where on admission the monophasic action potential duration and the effective refractory period were shortest and when the dispersion of the effective refractory period in the right ventricle was $80 \mathrm{~ms}$. However, two weeks after admission, the dispersion of the effective refractory period decreased to $20 \mathrm{~ms}$ and multiform ventricular tachycardia could not be induced.

Multiform ventricular tachycardia always followed the same $R$ on $T$ type ventricular extrasystoles in this patient. These ventricular extrasystoles showed a left bundle branch block with left axis deviation and seemed to originate from the right ventricular apex, where the monophasic action potential duration and the effective refractory period were shortest.

In patients with torsades de pointes, the dispersion of repolarisation is caused by prolongation of action potential durations in local areas of the ventricle and QT intervals are prolonged. In our patient, however, dispersion of repolarisation was caused by shortening of the monophasic action potential duration and the effective refractory period. The cause of the shortened effective refractory period at the right ventricular apex in this patient on admission is not certain. Acute myocardial ischaemia is known to shorten regional repolarisation, ${ }^{14}$ and ischaemic heart disease is reported to be associated with multiform ventricular tachycardia without QT prolongation $^{11516}$; but no ST change suggesting ischaemia was found on the electrocardiogram.

1 Soffer J, Dreifus LS, Michelson EL. Polymorphous ventricular tachycardia associated with normal and long QT intervals. Am f Cardiol 1982;49:2021-9.

2 Horowitz LN, Greenspan AM, Spielman SR, Josephson ME. Torsades de pointes: Electrophysiologic studies in patients without transient pharmacologic or metabolic patients without transient pharmacologic
abnormalities. Circulation 1981;63:1120-8.

3 Jackman WM, Friday KJ, Anderson JL, Aliot EM, Clark M, Lazzara R. The long QT syndromes: A critical review, new clinical observations and a unifying hypothesis. Prog Cardiovasc Dis 1988;31:115-72.

4 Roden DM, Hoffman BF. Action potential prolongation and induction of abnormal automaticity by low quinidine concentrations in canine Purkinje fibers: Relationship to potassium and cycle length. Circ Res 1985;56:857-67.

5 El-Sherif N, Zeiler RH, Craelius W, Gough WB, Henkin R. QTU prolongation and polymorphic ventricular tachyarrhythmias due to bradycardia-dependent early afterdepolarizations: Afterdepolarizations and ventricu-
lar arrhythmias. Circ Res 1988;63:286-305. 6 Surawicz B. Electrophysiologic substrate of torsade de pointes: dispersion of repolarization or early afterdepoarization? f Am Coll Cardiol 1989;14:172-84

7 Franz MR. Long-term recording of monophasic action potentials from human endocardium. Am $\mathcal{F}$ Cardiol 1983;51:1629-34.

8 Brachmann J, Scherlag BJ, Rosenshtraukh LV, Lazzara R. Bradycardia-dependent triggered activity: relevance to drug-induced multiform ventricular tachycardia. Circulation 1983;68:846-56.

9 El-Sherif N, Bekheit S, Henkin R. Quinidine-induced long QTU interval and torsades de pointes: Role of bradycardia-dependent early afterdepolarizations. $\mathscr{f} A m$ Coll Cardiol 1989;14:252-7.

10 Shimizu W, Tanaka K, Suenaga K, Wakamoto A Bradycardia-dependent early afterdepolarizations in a patient with QTU prolongation and torsades de pointes in associated with marked bradycardia and in associated with marked brady

11 Gavrilescu S, Luca C. Right ventricular monophasic action potentials in patients with long QT syndrome. $\mathrm{Br}$ Heart $\mathcal{f} 1978 ; 40: 1014-8$.

12 Shimizu W, Ohe T, Kurita T, Takaki H, Aihara N, Kamakura $\mathrm{S}$, et al. Early afterdepolarizations induced by isoproterenol in patients with congenital long QT syndrome. Circulation 1991;84:1915-23.

13 Kuo C, Munakata K, Reddy CP, Surawicz B. Characteristics and possible mechanism of ventricular arrhythmia dependent on the dispersion of action potential durations. Circulation 1983;67:1356-1367.

14 Surawicz B. Ventricular fibrillation. $\mathcal{f} \mathrm{Am}$ Coll Cardiol 1985;5:43B-54B.

15 Nguyen PT, Sheinman MM, Seger J. Polymorphous ventricular tachycardia: clinical characterization, therapy and the QT interval. Circulation 1986;74:340-9.

16 Zilcher H, Glogar D, Kaindl F. Torsades de pointes: occurrence in myocardial ischemia as a separate entityMultiform ventricular tachycardia or not? Eur Heart $f$ 1980;1:63-71. 